\title{
The time and space characteristics of magnetomotive force in the cascaded linear induction motor
}

\author{
Dajing Zhou • Jiaqing Ma $\cdot$ Lifeng Zhao \\ Xiao Wan · Yong Zhang • Yong Zhao
}

Received: 4 July 2013/Revised: 25 July 2013/Accepted: 2 August 2013/Published online: 7 September 2013

(C) The Author(s) 2013. This article is published with open access at Springerlink.com

\begin{abstract}
To choose a reasonable mode of three-phase winding for the improvement of the operating efficiency of cascaded linear induction motor, the time and space characteristics of magnetomotive force were investigated. The ideal model of the cascaded linear induction motor was built, in which the B and C-phase windings are respectively separated from the A-phase winding by a distance of $d$ and $e$ slots pitch and not overlapped. By changing the values of $d$ and $e$ from 1 to 5 , we can obtain 20 different modes of three-phase winding with the different combinations of $d$ and $e$. Then, the air-gap magnetomotive forces of A-, B-, and $\mathrm{C}$-phase windings were calculated by the magnetomotive force theory. According to the transient superposition of magnetomotive forces of A-, B-, and C-phase windings, the theoretical and simulated synthetic fundamental magnetomotive forces under 20 different arrangement modes were obtained. The results show that the synthetic magnetomotive force with $d=2$ and $e=4$ is close to forward sinusoidal traveling wave and the synthetic magnetomotive force with $d=4$ and $e=2$ is close to backward sinusoidal traveling wave, and their amplitudes and wave velocities are approximately constant and equal. In both cases, the motor could work normally with a
\end{abstract}

D. Zhou $(\bowtie) \cdot$ J. Ma $\cdot$ L. Zhao $\cdot$ X. Wan · Y. Zhang $\cdot$ Y. Zhao Key Laboratory of Magnetic Levitation Technologies and Maglev Trains (Ministry of Education of China), Southwest Jiaotong University, Chengdu 610031, Sichuan, China e-mail: zdj007008009@163.com

D. Zhou $\cdot$ J. Ma $\cdot$ L. Zhao $\cdot$ X. Wan $\cdot$ Y. Zhang $\cdot$ Y. Zhao Superconductivity and New Energy R\&D Center, Southwest Jiaotong University, Chengdu 610031, Sichuan, China

Y. Zhao

School of Materials Science and Engineering, University of New South Wales, Sydney, NSW 2052, Australia high efficiency, but under other 18 arrangement modes $(d=1, e=2 ; d=1, e=3 ; d=1, e=4 ; \ldots)$, the synthetic magnetomotive force presents obvious pulse vibration and moves with variable velocity, which means that the motor did not work normally and had high energy loss.

Keywords Linear induction motor .

Three-phase winding $\cdot$ Magnetomotive force

\section{Introduction}

Linear motor is a kind of electrical equipment which can directly convert the electrical energy to linear movement. Compared with traditional rotating machine, the drive system of linear motor works without the intermediate gearing, which simplifies the driver system, and makes its linear velocity unlimited and moving process without mechanical touch. In addition, the noise level of linear motor is very low. For all these characteristics, linear motor is widely applied to high-speed ground transportation [1-4].

When the pole number of a linear motor is not less than six, the values of negative-sequence current and zerosequence current are much small compared with the positive sequence current; thus their influence on the cascaded linear motor can be ignored [5, 6]. In this case, the asymmetry of three-phase current will not be considered. With three-phase symmetrical current flowing into threephase winding, linear motor will produce a traveling magnetomotive force (MMF) wave in the air-gap between primary and secondary windings. Since the energy exchange in mechanic-electronics is achieved by the airgap magnetic field of linear motor [7], the time and space characteristics of the MMF wave directly affect the operating efficiency and energy consumption in linear motor. 
The motor can work efficiently with the waveform of the MMF close to a sinusoidal traveling wave [8].

The finite element method (FEM) is an effective and accurate method to investigate the linear motor characteristics. Lu et al. [9] analyzed the features of air-gap magnetic field in large air-gap linear induction motor. Selcuk and Kurum [10] built a simplified FEM model of an actual short primary linear induction motor and solved it for airgap magnetic field distribution. Lu et al. [11] analyzed the two-dimensional transient air-gap magnetic field of long primary induction motor with the help of FEM. Li et al. [12] studied the characteristics of temperature field for tubular linear motor with the FEM. In this paper, we will solve the single-phase winding MMF and three-phase winding-synthesized MMFs in single-side linear induction motor (SLIM) by the classical theory of MMF [13] and adopt the FEM to validate the theoretical results. The work conditions and efficiency of SLIM are then optimized by analyzing MMF characteristics with different three-phase winding arrangement.

\section{Theoretical analysis and simulation model for SLIM}

\subsection{The MMF of A-phase winding}

The established theoretical model of SLIM is shown in Fig. 1, in which the leak flux in this model is ignored. In this model, it is assumed that primary and secondary are infinite long, primary iron yoke is not in magnetic saturation, the magnetoconductivity of primary and secondary is infinite, and magnetic induction intensity only contains the component in the $y$ axis direction. It is also assumed that the current flows along the $z$ axis direction, the equivalent air-gap $\delta$ between primary and secondary is distributed evenly along the $x$ axis direction, the magnetic potential difference of three-phase winding is distributed evenly along the air-gap, the number of pole pairs is $p$ and pole pitch is three slots pitch long, and the three-phase wingding is a bi-layered full-pitch winding.
Without loss of generality, the sinusoidal current as shown in Eq. (1) is assumed flowing into A-phase wingding, i.e.,

$i_{\mathrm{A}}=\sqrt{2} I_{\mathrm{A}} \cos \omega t$.

With reference to Fig. 1, according to the Ampere's circuital Law, A-phase winding MMF in air-gap can be expressed as [14]

$f_{\mathrm{A}}=H_{\mathrm{y}} \cdot \delta=N \cdot i_{\mathrm{A}} \quad$ when $-\frac{\pi}{2} \pm 2 m \pi \leq \theta \leq \frac{\pi}{2} \pm 2 m \pi$,

$f_{\mathrm{A}}=-H_{\mathrm{y}} \cdot \delta=-N \cdot i_{\mathrm{A}} \quad$ when $\frac{\pi}{2} \pm 2 m \pi \leq \theta \leq \frac{3 \pi}{2} \pm 2 m \pi$,

where $m=1,2,3, \ldots$, electrical angle $\theta=\pi x / \tau$, the number of turns in series winding $N=2 p N_{\mathrm{c}}$, and $N_{\mathrm{c}}$ is the number of turns in a single coil.

Since the quantity $f_{\mathrm{A}}(\theta)$ represents a periodic square wave along the air-gap, it can be represented by the Fourier series as

$f_{\mathrm{A}}(\theta)=f_{\mathrm{A} 1} \cos \theta+f_{\mathrm{A} 3} \cos 3 \theta+f_{\mathrm{A} 5} \cos 5 \theta+\cdots$ $+f_{\mathrm{A} n} \cos n \theta$,

where $f_{\mathrm{A} n}$ is

$f_{\mathrm{A} n}=\frac{4}{T} \int_{0}^{\frac{T}{2}} f_{\mathrm{A}}(\theta) \cos \left(n \omega_{0} \theta\right) \mathrm{d} \theta=\frac{1}{n} \cdot \frac{4}{\pi} \cdot N i_{\mathrm{A}} \sin n \frac{\pi}{2}$,

$n=1,2,3, \ldots$

Substituting Eqs. (1) and (4) into Eq. (3), we have the instantaneous value of A-phase wingding MMF:

$$
\begin{aligned}
f_{\mathrm{A}}(\theta, t)= & \frac{4 \sqrt{2}}{\pi} N I_{A}\left[\cos \theta-\frac{1}{3} \cos 3 \theta+\frac{1}{5} \cos 5 \theta\right. \\
& \left.-\cdots+\frac{1}{n} \sin \left(n \frac{\pi}{2}\right) \cos n \theta\right] \cos \omega t .
\end{aligned}
$$

From Eq. (5), we can find that A-phase winding MMF after the Fourier series transformation can be decomposed

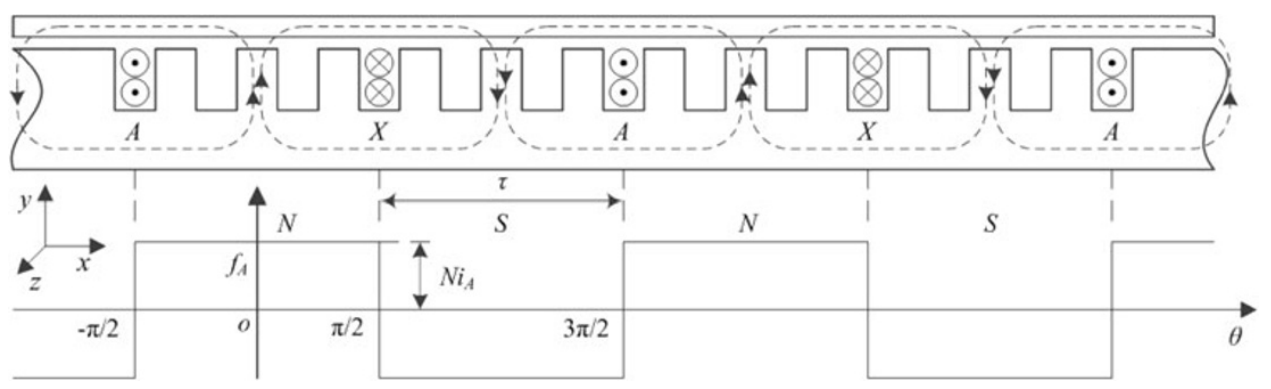

Fig. 1 The theoretical model of SLIM and A-phase winding MMF distribution diagram 
into fundamental wave and a series of higher harmonics, and the amplitudes of fundamental wave and higher harmonics pulse over time with the current frequency. Because fundamental wave determines the energy conversion of linear motor and its main performance, it is most important and fundamental to analyze the fundamental MMF [15].

\subsection{The MMFs of three-phase winding}

As shown in Fig. 2, when the B and C-phase windings are respectively $d$ and $e$ slots pitch away from the A-phase winding, we can obtain the MMF of B-phase winding and C-phase winding respectively by moving the MMF of Aphase winding $\pi d / 3$ and $\pi e / 3$ along the positive direction of $\theta$ axis, respectively.

Let three-phase current flow into three-phase winding, through above analysis we can obtain the expression of the fundamental MMF for A, B, C phase windings, respectively,

$F_{\mathrm{A}}(\theta, t)=\frac{4 \sqrt{2}}{\pi} N I_{\mathrm{A}} \cos (\theta) \cos (\omega t)$,

$F_{\mathrm{B}}(\theta, t, d)=\frac{4 \sqrt{2}}{\pi} N I_{\mathrm{A}} \cos \left(\theta-\frac{1}{3} \pi d\right) \cos \left(\omega t-\frac{2}{3} \pi\right)$,

$F_{\mathrm{C}}(\theta, t, e)=\frac{4 \sqrt{2}}{\pi} N I_{\mathrm{A}} \cos \left(\theta-\frac{1}{3} \pi e\right) \cos \left(\omega t+\frac{2}{3} \pi\right)$.

Adding the instantaneous values of fundamental MMF with $\mathrm{A}, \mathrm{B}, \mathrm{C}$ phase windings, we have the fundamental MMFs:

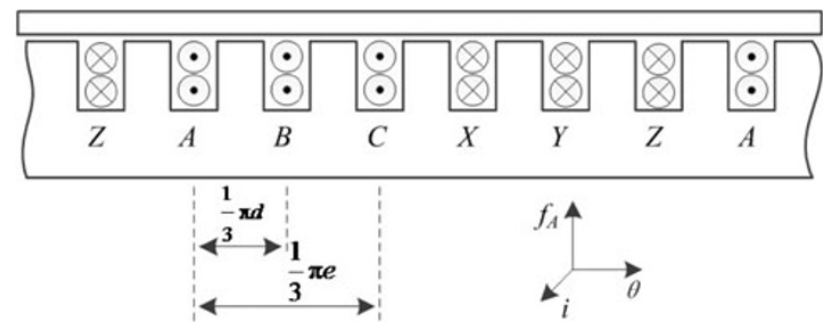

Fig. 2 Three-phase winding distribution diagram
$F(\theta, t, d, e)=F_{\mathrm{A}}(\theta, t)+F_{\mathrm{B}}(\theta, t, d)+F_{\mathrm{C}}(\theta, t, e)$.

According to the theoretical model of SLIM, the values of $d$ and $e$ can be taken from 1,2,3, 4 and 5 in a cycle of MMF, so that the number of arrangement modes of threephase winding is 20 with the combination between $d$ and $e$. The results of fundamental $\mathrm{MMF}_{\mathrm{S}}$ with different arrangements of three-phase windings will be analyzed later.

\subsection{The simulation model for SLIM}

The simulation model of SLIM is established by Ansoft Maxwell as shown in Fig. 3. Table 1 presents the specific parameters related to this model. Different simulation data of MMFs will be obtained by changing the relative positions of three-phase winding.

\section{Results and analysis}

3.1 The MMFs with $d=1$ and $e=2$

Referring to Fig. 2, we move B-phase winding and C-phase winding from the position of A-phase winding 1 slot pitch and 2 slots pitch, respectively. Then, substitute $d=1$ and $e=2$ into Eq. (7) and the expression of fundamental MMFs is calculated by trigonometric formula [16]:

$$
\begin{aligned}
F(\theta, t, 1,2)= & {\left[\frac{2 \sqrt{2}}{\pi} N I_{\mathrm{A}}[2 \cos (\omega t+\theta)+\cos (\omega t-\theta)\right.} \\
& +\cos (\omega t+\theta-\pi)+\cos \left(\omega t-\theta-\frac{1}{3} \pi\right) \\
& \left.+\cos \left(\omega t-\theta+\frac{4}{3} \pi\right)\right]
\end{aligned}
$$

The MMF is composed of three forward traveling waves and two back traveling waves referring to Eq. (8), and the results are shown in Fig. 4. As can be seen in Fig. 4a, when the spatial MMF waveform is at the transient time of $\omega t=0, \pi / 2, \pi, 3 \pi / 2$, respectively, and with a scaling factor $\beta=2 \sqrt{2} N I_{\mathrm{A}} / \pi$, the MMF pushes to the left in space and exists obvious vibration. The movement with variable velocity is shown in Fig. 4c, d. These features will reduce

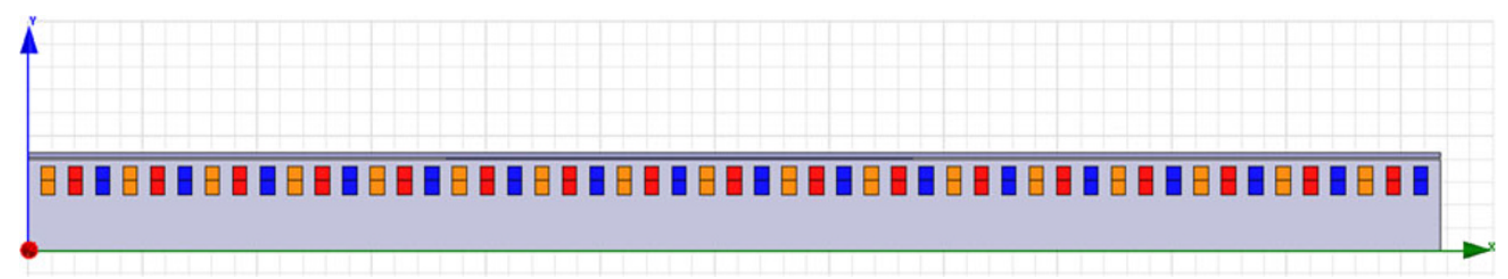

Fig. 3 The simulation model of SLIM 
the efficiency of linear motor thrust in the horizontal direction, and at the same time also bring high energy loss.

According to the simulation model for SLIM, when arranging three-phase winding to be $d=1$ and $e=2$, the simulation transient waveform of the MMF is shown in Fig. $4 \mathrm{~b}$, which further validates the characteristics of MMF with $d=1$ and $e=2$.

3.2 The MMFs with $d=2, e=4$, and $d=4, e=2$

In order to keep the amplitude and wave velocity of MMF invariant over time and avoid the pulse vibration and

Table 1 SLIM parameters for simulation model

\begin{tabular}{llll}
\hline Parameter & Value & Parameter & Value \\
\hline Virtual current $I_{\mathrm{A}}$ & $8 \mathrm{~A}$ & Air-gap $\delta$ & $0.5 \mathrm{~mm}$ \\
Power frequency $f$ & $50 \mathrm{~Hz}$ & Slots per pole per phase $q$ & 1 \\
Pole pitch $\tau$ & $36 \mathrm{~mm}$ & Turns per coil $N_{\mathrm{c}}$ & 60 \\
Pole pairs $p$ & 8 & & \\
\hline
\end{tabular}
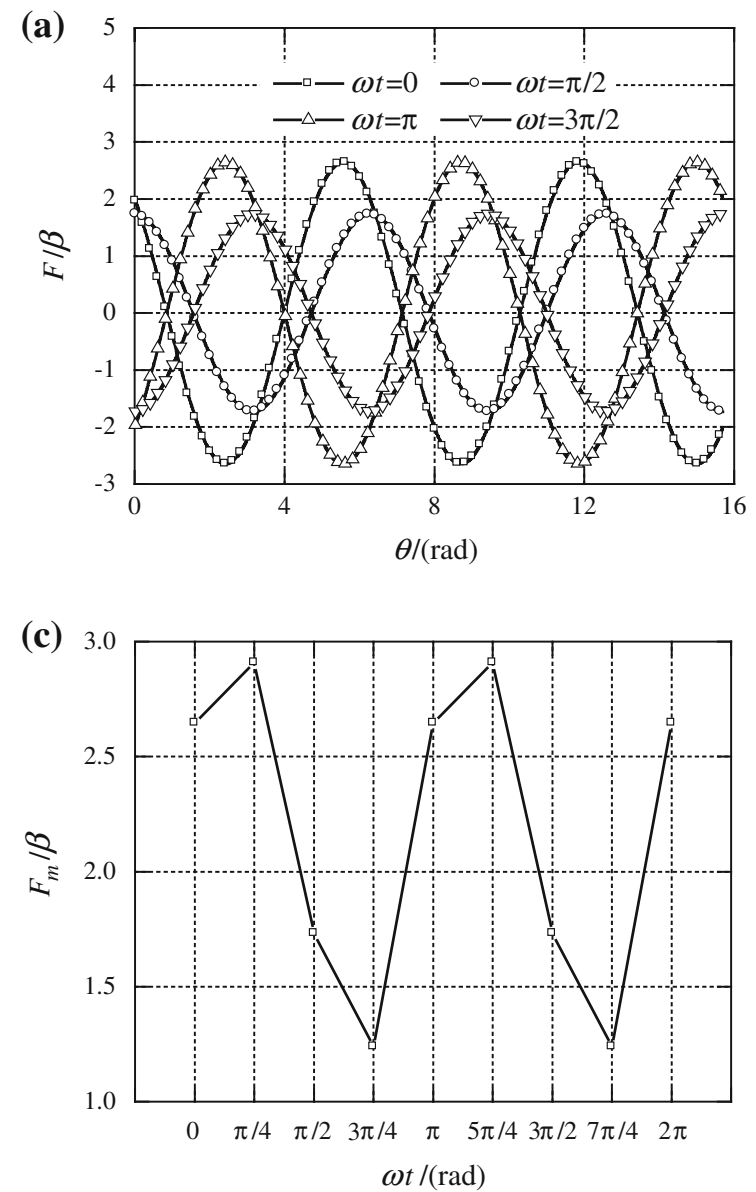

movement with variable velocity of MMF, we solved the MMFs under different arrangements of three-phase winding. The result indicates that the MMFs in the condition of $d=2, e=4$ or $d=4, e=2$ are a traveling wave with its amplitude and velocity being constant as shown in Fig. 5a, c, which are expressed, respectively, by Eqs. (9a) and (9b):

$F(\theta, t, 2,4)=\frac{6 \sqrt{2}}{\pi} N I_{\mathrm{A}} \cos (\omega t-\theta)$

$F(\theta, t, 4,2)=\frac{6 \sqrt{2}}{\pi} N I_{A} \cos (\omega t+\theta)$,

where $\theta=k \cdot x=\frac{\pi}{\tau} \cdot x$.

From Fig. 5a, c, we can see that $F(\theta, t, 2,4)$ is a forward traveling wave and $F(\theta, t, 4,2)$ is a backward traveling wave. Their amplitude and wave velocity can be expressed as

$$
\begin{aligned}
& F_{\mathrm{m}}(\theta, t, 2,4)=F_{\mathrm{m}}(\theta, t, 4,2)=\frac{6 \sqrt{2}}{\pi} N I_{\mathrm{A}}, \\
& v(\theta, t, 2,4)=v(\theta, t, 4,2)=\frac{\omega}{k}=\frac{\tau \cdot \omega}{\pi} .
\end{aligned}
$$
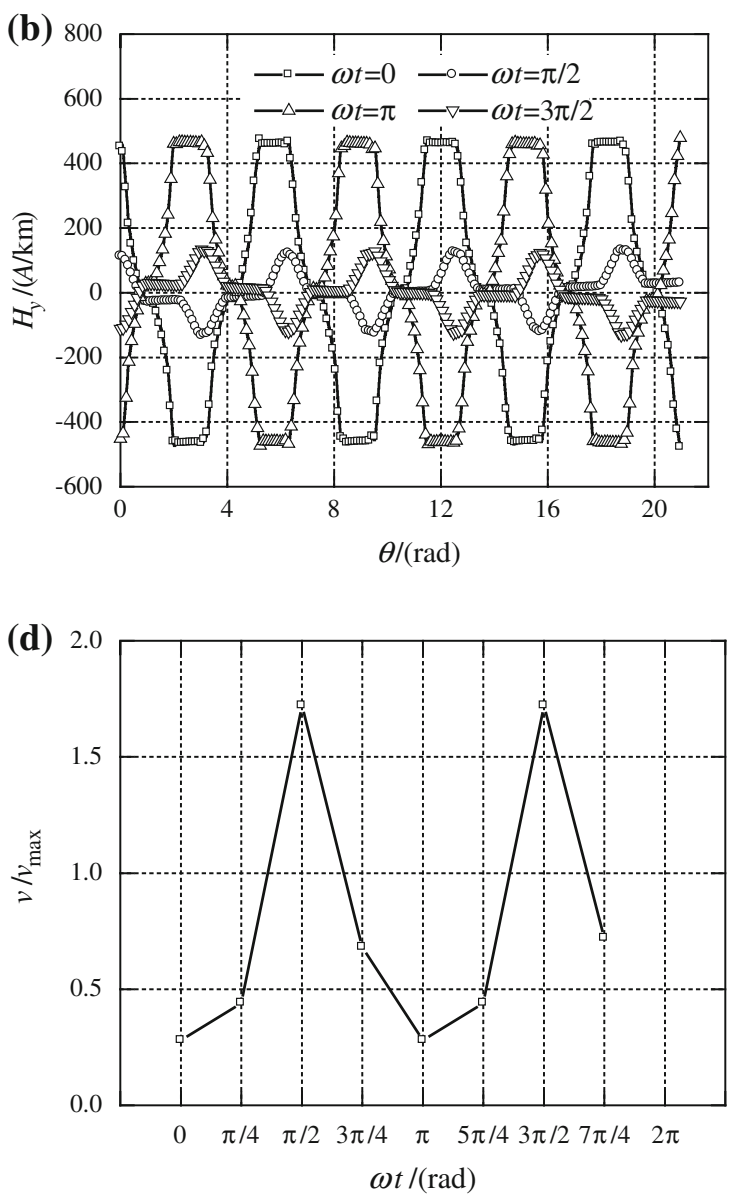

Fig. 4 The MMFs with $d=1$ and $e=2$ and its amplitude and velocity characteristics. a theoretical fundamental MMFs. b simulated MMFs. c amplitude of theoretical fundamental MMF. d velocity of theoretical fundamental MMF 

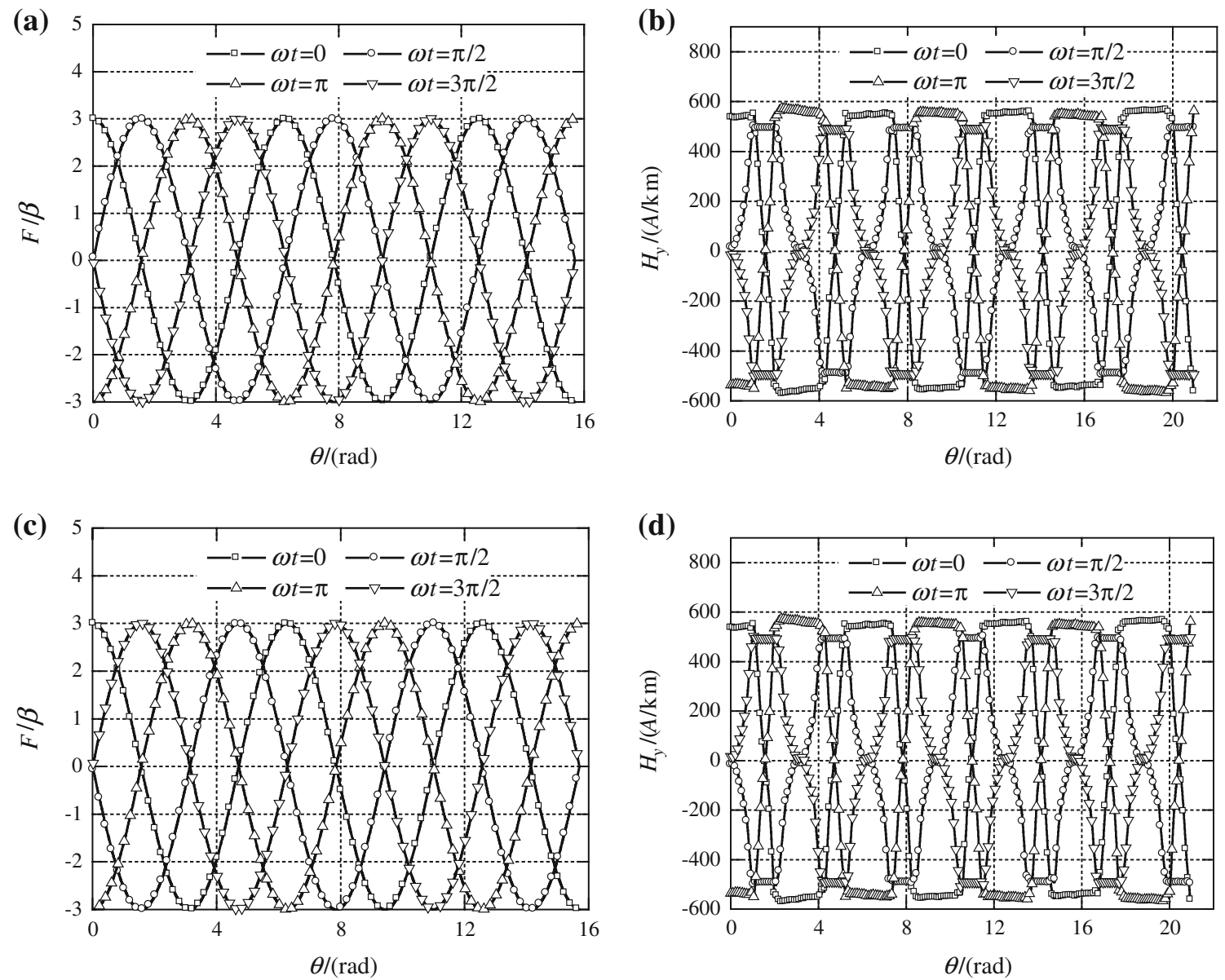

Fig. 5 The MMFs with $d=2, e=4$ and $d=4, e=2$. a theoretical fundamental MMFs with $d=2$ and $e=4$. b simulated MMFs with $d=2$ and $e=4$. c theoretical fundamental MMFs with $d=4$ and $e=2$. d simulated MMFs with $d=4$ and $e=2$

Based on the simulation model for SLIM, we also changed the arrangement mode of three-phase winding to $d=2, e=4$ and $d=4, e=2$, respectively, and obtained the MMFs simulated by Ansoft Maxwell. The results are shown in Fig. 5b, d. Since the simulation model for SLIM is not fully ideal and its MMFs contain other higher harmonics, the MMFs are close to sinusoidal traveling wave with constant amplitude and velocity.

\section{Conclusion}

Based on the classical MMF theory of rotating machine, we established the theoretical model and simulation model for SLIM, in which three-phase winding has 20 arrangement modes in a cycle of MMF, and the arrangement mode determines the time and space characteristics of MMFs. Through the calculation with electromagnetic theory and finite element software simulation, we solved and discussed the MMFs under 20 arrangement modes of three-phase winding, respectively. On the basis of the above analysis results, the conclusions are drawn as follows:

(1) The MMFs with $d=2$ and $e=4$ are close to sinusoidal wave, and it travels toward the positive direction with the constant amplitude and velocity. In this case, the motor can work normally with a high efficiency.

(2) The MMFs with $d=4$ and $e=2$ are close to sinusoidal wave, and travel toward the negative direction with the constant amplitude and velocity. Also the motor can work normally with a high efficiency.

(3) In other 18 conditions, the MMFs show obvious pulse vibration and movement with variable velocity, such as the MMFs under $d=1, e=2$. This means that the motor does not work normally, and instead it has high energy loss.

Acknowledgments This work was supported by the National Magnetic Confinement Fusion Science Program 2011GB112001, 
Program of International S\&T Cooperation S2013ZR0595, the financial support of the National Natural Science Foundation of China (No. 51271155), the Fundamental Research Funds for the Central Universities (SWJTU11ZT16, SWJTU11ZT31), the Science Foundation of Sichuan Province 2011JY0031, 2011JY0130.

Open Access This article is distributed under the terms of the Creative Commons Attribution License which permits any use, distribution, and reproduction in any medium, provided the original author(s) and the source are credited.

\section{References}

1. Ye YY (2000) Principle and application of linear motor. China Machine Press, Beijin (in Chinese)

2. Wang TC (1971) Linear induction motor for high-speed ground transportation. IEEE Trans Ind Gen Appl 7(5):632-642

3. Deng JM, Chen TF, Tang JX et al (2013) Optimum slip frequency control of Maglev single-sided linear induction motors to maximum dynamic thrust. Proc CSEE 33(12):123-130 (in Chinese)

4. Wang K, Shi LM, He JW et al (2009) A decoupling control of normal-and-thrust forces in single-sided linear induction motor. Proc CSEE 29(6):100-104 (in Chinese)

5. Lu JY, Ma WM, Sun ZL et al (2009) Research on static longitudinal end effect of linear induction motor with multi-segment primary. Proc CSEE 29(33):95-101 (in Chinese)

6. Sun ZL, Ma WM, Lu JY et al (2010) Research of static longitudinal end effect and impedance matrix for long primary double- sided linear induction motors. Proc CSEE 30(18):72-77 (in Chinese)

7. Long LX (2006) Theory of linear induction motor and its electromagnetic design method. Science Press, Beijing (in Chinese)

8. Fan SD (1996) Determination for magnetic density wave in the gap of the permanent magnet DC motor. Small Spec Electr Mach 3:2-5 (in Chinese)

9. Lu QF, Fang YT, Ye YY (2005) A study on force characteristic of large air gap linear induction motor. Proc CSEE 25(21):132136 (in Chinese)

10. Selcuk AH, Kurum $H$ (2008) Investigation of end effects in linear induction motors by using the finite-element method. IEEE Trans Magn 44(7):1791-1795

11. Lu JY, Ma WM, Xu J (2008) Modeling and simulation of high speed long primary double-sided linear induction motor. Proc CSEE 28(27):89-94 (in Chinese)

12. Li LY, Huang XZ, Kou BQ et al (2013) Numerical calculation of temperature field for tubular linear motor based on finite element method. Trans China Electrotech Soc 28(2):132-138 (in Chinese)

13. Lipo TA (2004) Introduction to AC machine design. University of Wisconsin Press, Chicago

14. Liu CY, Wang H, Zhang ZJ et al (2011) Research on thrust characteristics in permanent magnet linear synchronous motor based on analysis of nonlinear inductance. Proc CSEE 31(30):6976 (in Chinese)

15. Xie MT, Zhang GY (2004) Electromechanics. University of Chongqing Press, Chongqing (in Chinese)

16. Zhou SL (2003) The analysis of stator magnetic potential in the three-phase asynchronous motor. J Tongling Coll 2(2):73-74 (in Chinese) 